den hergestellten Kalligrafien überwiegend ein hohes $M a ß$ an eigenschöpferischen Inhalten zukommt, sodass sie als von dem zugrunde liegenden Schriftzeichen losgelöstes eigenes künstlerisches Werk und nicht als bloß handwerkliche Wiedergabe eines in der kulturellen Tradition wurzelnden Schriftzeichens zu bewerten ist; dies ist ggf. durch eine sachverständige Begutachtung zu klären. Liegt der Schwerpunkt der Tätigkeit hingegen nur auf der handwerklichtechnischen Bearbeitung von Materialien wie Holz, Metall, Stein o. ä. oder auf der Herstellung von Gebrauchstexten auf Papier, kommt eine Einordnung der Kalligrafie als Kunst iSd § 2 Satz 1 KSVG nur dann in Betracht, wenn der Urheber trotz seiner Stellung als Kunsthandwerker mit seinen Werken in Kunstkreisen als Künstler anerkannt und behandelt wird (vgl. BSGE 80, 136, $138=$ SozR 3-5425 § 2 Nr. 5; BSGE 82, $164=$ SozR 3-5425 § 2 Nr. 8). In diesem Fall ist vor allem maßgebend, ob der Betroffene an Kunstausstellungen teilnimmt, Mitglied von Künstlervereinen ist, in Künstlerlexika aufgeführt wird, Auszeichnungen als Künstler erhalten hat oder andere Indizien auf seine Anerkennung als Künstler schließen lassen.

Nach den mit der Sprungrevision nicht angreifbaren und deshalb für den Senat bindenden ( $§ 161$ Abs. 4, § 163 SGG) Feststellungen des SG ist der Kläger Künstler im Sinne des KSVG. Die von inm geschaffenen Kalligrafien gehen über die Wiedergabe traditionellen Kulturgutes hinaus. Der Kläger wandelt das jeweilige arabische Schriftzeichen individuell so ab, dass es für den Betrachter nicht mehr eindeutig erkennbar ist. Durch seine kreative Gestaltgebung wird das Schriftzeichen entfremdet und das neu geschaffene Bild oder die neu geschaffene Grafik mit einer eigenständigen künstlerischen Aussage versehen, die über das reine Schönschreiben eines Buchstabens oder Zeichens weit hinaus geht. Der Kläger schafft Werke mit eigener Identität, die sich nicht von denen eines herkömmlichen Malers oder künstlerischen Grafikers unterscheiden.

Er gehört deshalb zu dem durch das KSVG geschützten Personenkreis und ist zumindest seit 2003 Künstler iSd § 2 Satz 1 KSVG. Es kommt deshalb nicht darauf an, ob er an Kunstausstellungen teilnimmt, Mitglied von Künstlervereinen ist oder ob er als Künstler in bestimmten Kunstkreisen Anerkennung gefunden hat. Diese vom SG zusätzlich angestellten Erwägungen wären nur dann erforderlich, wenn der Kläger überwiegend (kunst) handwerklich tätig wäre. Dies ist nach den Feststellungen des SG aber gerade nicht der Fall.

\title{
Kunsthändler und Folgerecht
}

Bundesgerichtshof, Urteil vom 17. Juli 2008 - I ZR 109/05

1. Kunsthändler iSd § 26 UrhG ist jeder, der aus eigenem wirtschaftlichem Interesse an der Veräußerung von Kunstwerken beteiligt ist. Hierzu zählt auch, wer Sammler und Kunstinteressenten beim Kauf und Verkauf von Kunstwerken berät und hierfür eine von der Höhe des Kaufpreises abhängige Provision beansprucht.

2. Der Auskunftsanspruch des Künstlers gegen den Kunsthändler oder Versteigerer gemäß § 26 Abs. 4 Satz 1 UrhG (F: 10.11.1972) setzt ebenso wie der Folgerechtsanspruch des Künstlers gegen den Veräußerer gemäß § 26 Abs. 1 Satz 1 UrhG (F: 10.11.1972) voraus, dass die Weiterveräußerung zumindest teilweise im Inland erfolgt ist.

3. Unter Weiterveräußerung iSd § 26 UrhG ist nicht allein das dingliche Verfügungsgeschäft, sondern das gesamte, das schuldrechtliche Verpflichtungsgeschäft ebenso wie das dingliche Verfügungsgeschäft umfassende Veräußerungsgeschäft zu verstehen (im Anschluss an BGHZ 126, 252, 259 [BGH 16.06.1994 - I ZR 24/92] - Folgerecht bei Auslandsbezug).

4. Bei Unterzeichnung des Kaufvertrags durch einen Vertragspartner im Inland ist der erforderliche Inlandsbezug gegeben. 5. Die Bestimmung des $§ 167$ ZPO ist grundsätzlich auch in den Fällen anwendbar, in denen durch die Zustellung eine Frist gewahrt werden soll, die auch durch außergerichtliche Geltendmachung gewahrt werden kann (Abgrenzung zu BGH, Urt. v. 11.10.1974 - VZR 25/73, NJW 1975, 39; Aufgabe von BGH, Urt. v. 10.2.1971 - VIII ZR 208/69, WM 1971, 383, 384 und Urt. v. 21.10.1981 - VIII ZR 212/80, NJW 1982, 172).

\section{Tatbestand}

- Die Klägerin ist die Verwertungsgesellschaft Bild-Kunst. Sie nimmt in Deutschland die urheberrechtlichen Befugnisse der ihr angeschlossenen Urheber an Werken der bildenden Künste wahr; hierzu gehört auch der Folgerechtsanspruch nach § 26 UrhG. Der Beklagte berät gegen Provision Sammler und Kunstinteressenten beim Kauf und Verkauf von Kunstwerken.
Die Klägerin verlangt vom Beklagten Auskunft über die Weiterveräußerung von Originalwerken der bildenden Künste ihr angeschlossener Urheber. Sie begehrt zum einen allgemein Auskunft darüber, welche Werke unter seiner Beteiligung im Jahre 2001 weiterveräußert wurden (§ 26 Abs. 3 UrhG a.F.). Sie erstrebt zum anderen nähere Auskunft über die Veräußerung der Kunstsammlung Ahlers im Januar 2001 und möchte insoweit den Namen und die Anschrift des Veräußerers sowie die Höhe des Veräußerungserlöses der einzelnen Werke erfahren (§ 26 Abs. 4 UrhG a.F.). 
Die „Sammlung Ahlers" war eine der größten Privatsammlungen des Expressionismus mit Werken der Künstler des „Blauen Reiter" und der „Brücke“. Sie enthielt zahlreiche Werke, bei denen die Schutzdauer des Urheberrechts noch nicht abgelaufen war. Die Verkäufer, zu denen jedenfalls die Ahlers AG und weitere Unternehmen der Ahlers-Gruppe gehören, haben den Kaufvertrag am 26. Januar 2001 in Frankfurt am Main unterschrieben. Im Übrigen sind die Umstände des Abschlusses und der Durchführung des Vertrages streitig, insbesondere ist streitig, in welcher Weise der Beklagte an diesem Geschäft beteiligt war und ob die Kunstwerke sich bereits bei Vertragsschluss in einem Zollfreilager in der Schweiz befanden.

Der Beklagte ist der Klage entgegengetreten.

Das Landgericht hat dem allgemeinen Auskunftsanspruch stattgegeben und den die "Sammlung Ahlers" betreffenden Auskunftsanspruch abgewiesen. Das Berufungsgericht hat den allgemeinen Auskunftsanspruch abgewiesen und dem die „Sammlung Ahlers" betreffenden Auskunftsanspruch stattgegeben (OLG Frankfurt GRUR 2005, 1034).

Mit der vom Berufungsgericht zugelassenen Revision verfolgt die Klägerin den allgemeinen Auskunftsanspruch weiter, während der Beklagte die Abweisung des die „Sammlung Ahlers" betreffenden Auskunftsanspruchs erstrebt. [...]

\section{Entscheidungsgründe}

A. Das Berufungsgericht hat den Auskunftsanspruch der Klägerin hinsichtlich der "Sammlung Ahlers" bejaht und den allgemeinen Auskunftsanspruch mangels rechtzeitiger Geltendmachung abgewiesen. Zur Begründung hat es ausgeführt: Der Auskunftsanspruch hinsichtlich der Veräußerung der „Sammlung Ahlers" sei nach § 26 Abs. 4 UrhG (a.F.) begründet. Der Beklagte sei als Kunsthändler iSv § 26 UrhG anzusehen. Kunsthändler im Sinne dieser Bestimmung sei auch der Kunstvermittler, der gegen Provision beim Kunsthandel berate. Der Beklagte sei an der Veräußerung der "Sammlung Ahlers" beteiligt gewesen. Er habe die Gemäldesammlung gemeinsam mit dem amerikanischen Kunsthändler N. zum Zweck der Weiterveräußerung erworben. Dass der Beklagte insoweit als Kunsthändler tätig geworden sei, erscheine auch nicht deshalb zweifelhaft, weil - nach seiner Behauptung - Erwerber der Sammlung eine aus ihm und seinem Partner N. bestehende amerikanische Partnership gewesen sei. Auch der für den Folgerechtsanspruch erforderliche Inlandsbezug der Weiterveräußerung sei gegeben. Die Klägerin habe vorgetragen, die Einigung über den Eigentumsübergang sei schon in dem in Deutschland unterzeichneten Kaufvertrag enthalten gewesen. Der Beklagte, den eine sekundäre Darlegungslast treffe, habe diese Behauptung nicht ausreichend substantiiert bestritten. Daher gelte das Vorbringen der Klägerin, wonach ein Teil des dinglichen Veräußerungsgeschäfts im Inland stattgefunden habe, als zugestanden. Der allgemeine Auskunftsanspruch nach § 26 Abs. 3 UrhG (a.F.) sei unbegründet. Die Klägerin habe nicht bewiesen, dass sie die allgemeine Auskunft für das Jahr 2001 spätestens bis zum 31. Dezember 2002 vom Beklagten verlangt habe. Das mit der am 10. Februar 2003 zugestellten Klageschrift geltend gemachte Auskunftsersuchen wirke nicht auf den Zeitpunkt der Einreichung der Klageschrift am 20. Dezember 2002 zurück. Die Bestimmung des § 167 ZPO gelte nicht für Fristen, die - wie hier - sowohl durch gerichtliche als auch durch außergerichtliche Geltendmachung gewahrt werden könnten.

B. Die gegen diese Beurteilung gerichteten Revisionen haben Erfolg und führen zur Aufhebung des Berufungsurteils. Auf die Revision der Klägerin ist das Urteil des Landgerichts wiederherzustellen, soweit dieses dem allgemeinen Auskunftsanspruch stattgegeben hat (dazu unter II). Auf die Revision des Beklagten ist die Sache zur neuen Verhandlung und Entscheidung über den die "Sammlung Ahlers" betreffenden Auskunftsanspruch an das Berufungsgericht zurückzuverweisen (dazu unter III).

I. Der Folgerechtsanspruch nach § 26 UrhG ist durch das Fünfte Gesetz zur Änderung des Urheberrechtsgesetzes vom 10. November 2006 (BGBI. I S. 2587) neu geregelt worden. Diese Neuregelung ist am 16. November 2006 in Kraft getreten. Für den Streitfall ist die zuvor geltende Rechtslage maßgeblich, da die Auskunftsansprüche vor dem Inkrafttreten der Neuregelung geltend gemacht worden sind.

II. Entgegen der Ansicht des Berufungsgerichts ist der allgemeine Auskunftsanspruch nach § 26 Abs. 3 UrhG a.F. begründet. Nach dieser Bestimmung kann der Urheber von einem Kunsthändler oder Versteigerer Auskunft darüber verlangen, welche Originale von Werken des Urhebers innerhalb des letzten vor dem Auskunftsersuchen abgelaufenen Kalenderjahres unter Beteiligung des Kunsthändlers oder Versteigerers weiterveräußert wurden.

1. Die Klägerin ist als Verwertungsgesellschaft nach § 26 Abs. 5 UrhG a.F. berechtigt, den Anspruch auf Auskunftserteilung hinsichtlich der ihr angeschlossenen Urheber geltend zu machen.

2. Der Beklagte ist, wie das Berufungsgericht zutreffend angenommen hat, Kunsthändler iSd § 26 UrhG und daher zur Auskunftserteilung verpflichtet.

a) Der Begriff des Kunsthändlers ist in einem weiten Sinne zu verstehen. Kunsthändler iSd § 26 UrhG ist jeder, der aus eigenem wirtschaftlichem Interesse an der Veräußerung von Kunstwerken beteiligt ist (vgl. Dreier/Schulze UrhG, 2. Aufl., § $26 \mathrm{Rn}$. 15; Fromm/Nordemann Urheberrecht, 9. Aufl., § 26 UrhG Rn. 4; Schricker/Katzenberger Urheberrecht, 3. Aufl., § 26 UrhG Rn. 33; Wandtke/Bullinger Urheberrecht, 2. Aufl., § 26 UrhG Rn. 12). Dabei kann die Beteiligung des Kunsthändlers, wie sich aus § 26 Abs. 1 Satz 1 UrhG ergibt, nicht nur darin bestehen, dass er Erwerber oder Veräußerer des Kunstwerks ist, sondern auch darin, dass er bei der Veräußerung des Kunstwerks als Vermittler tätig wird. Als Vermittler wird der Kunsthändler schon dann tätig, wenn er 
das Veräußerungsgeschäft zwischen dem Veräußerer und dem Erwerber fördert. Insoweit können bereits Hinweise auf das Kunstwerk, dessen Aufnahme in einen Katalog oder in Ausstellungen genügen (vgl. Dreier/Schulze aaO § 26 Rn. 16; Fromm/ Nordemann aaO § 26 UrhG Rn. 4; Möhring/Nicolini/Spautz UrhG, 2. Aufl., § 26 Rn. 11; Schricker/Katzenberger aaO § 26 UrhG Rn. 33; Wandtke/Bullinger aaO § 26 UrhG Rn. 13).

b) Nach diesen Maßstäben ist der Beklagte als Kunsthändler iSd § 26 UrhG anzusehen. Insoweit ist es nicht von Bedeutung, dass er selbst keine Kunstwerke ankauft oder verkauft und sich selbst nicht als Kunsthändler, sondern als Kunstberater bezeichnet. Seine Tätigkeit erschöpft sich nicht im Erstellen von Expertisen. Er berät Sammler und Kunstinteressenten beim Kauf und Verkauf von Kunstwerken. Damit fördert er die Veräußerung dieser Werke. Er hat ein eigenes wirtschaftliches Interesse am Zustandekommen von Veräußerungsgeschäften. Er erhält für seine Tätigkeit eine Provision, die nach den getroffenen Feststellungen in einem - stets vom Verkäufer zu zahlenden - Prozentsatz des Kaufpreises besteht.

3. Die Klägerin hat den Auskunftsanspruch entgegen der Ansicht des Berufungsgerichts rechtzeitig geltend gemacht.

a) Auskunft kann nach $\S 26$ Abs. 3 UrhG a.F. nur über Weiterveräußerungen innerhalb des letzten vor dem Auskunftsersuchen abgelaufenen Kalenderjahres verlangt werden. Der ein bestimmtes Kalenderjahr betreffende Auskunftsanspruch kann demnach nur bis zum Ablauf des folgenden Kalenderjahres geltend gemacht werden; das Auskunftsersuchen muss dem Kunsthändler oder Versteigerer daher spätestens bis zum letzten Tag des Folgejahres zugegangen sein (vgl. Fromm/Nordemann aaO $\S 26$ UrhG Rn. 6).

b) Die Klägerin hat den Anspruch auf Auskunftserteilung über die im Jahre 2001 weiterveräußerten Werke mit ihrer am 20. Dezember 2002 bei Gericht eingegangenen Klageschrift geltend gemacht, die dem Beklagten am 10. Februar 2003 zugestellt worden ist. Entgegen der Auffassung des Berufungsgerichts wirkt die Zustellung nach § 167 ZPO auf den Zeitpunkt des Eingangs der Klageschrift zurück. Damit ist das Auskunftsersuchen rechtzeitig zugegangen.

Soll durch die Zustellung eine Frist gewahrt werden, tritt diese Wirkung nach § 167 ZPO bereits mit Eingang des Antrags oder der Erklärung ein, wenn die Zustellung demnächst erfolgt. Diese Voraussetzungen sind hier erfüllt. Durch die Zustellung der Klageschrift sollte die Frist zur Geltendmachung des Auskunftsanspruchs nach § 26 Abs. 3 UrhG a.F. gewahrt werden. Die Klageschrift wurde "demnächst", also ohne der Klägerin zuzurechnende Verzögerungen im Zustellungsverfahren, zugestellt (vgl. BGHZ 168, 306, 310 ff.). Das Berufungsgericht hat gemeint, die Bestimmung des $\S 167$ ZPO gelte nicht für Fristen, die - wie hier die Frist zur Geltendmachung des Auskunftsanspruchs - sowohl durch gerichtliche als auch durch außergerichtliche Geltendmachung gewahrt werden könnten. Der Senat teilt diese Auffassung nicht. [...]
Nach Ansicht des Senats ist die Bestimmung des $\S 167$ ZPO grundsätzlich auch in den Fällen anwendbar, in denen durch die Zustellung eine Frist gewahrt werden soll, die auch durch außergerichtliche Geltendmachung gewahrt werden kann. [...]

Soweit der V. und der VIII. Zivilsenat in der Vergangenheit die Auffassung vertreten haben, eine Rückwirkung der Zustellung komme generell bei Fristen nicht in Betracht, die auch durch außergerichtliche Geltendmachung gewahrt werden könnten, haben sie auf Anfrage erklärt, an dieser Auffassung nicht festzuhalten (§ 132 Abs. 3 Satz 1 GVG). Der V. Zivilsenat schließt allerdings für die von inm entschiedene Frage der Wahrung der Anfechtungsfrist des § 121 BGB (BGH NJW 1975, 39 f.) eine Anwendung des § 167 ZPO nach wie vor aus: In diesem Fall komme das Interesse des Empfängers, rasch Klarheit darüber zu gewinnen, ob der Anfechtungsberechtigte von seinem Gestaltungsrecht Gebrauch mache, in dem Erfordernis der Unverzüglichkeit der Anfechtung zum Ausdruck und verbiete eine Rückwirkung der Zustellung.

III. Der die "Sammlung Ahlers" betreffende Auskunftsanspruch kann auf der Grundlage der bislang getroffenen Feststellungen nicht zugesprochen werden.

1. Zu Recht hat das Berufungsgericht allerdings angenommen, dass die Weiterveräußerung der "Sammlung Ahlers" den erforderlichen Inlandsbezug aufweist.

a) Der Auskunftsanspruch gemäß § 26 Abs. 4 Satz 1 UrhG a.F. setzt ebenso wie der Folgerechtsanspruch gemäß § 26 Abs. 1 Satz 1 UrhG a.F. nach dem im Urheberrecht geltenden Territorialitätsprinzip voraus, dass die Weiterveräußerung im Sinne dieser Vorschrift zumindest teilweise im Inland stattgefunden hat (vgl. BGHZ 126, 252, 254 ff. - Folgerecht bei Auslandsbezug; vgl. auch BGHZ 152, 317, 326 f. - Sender Felsberg; BGH, Urt. v. 24. Mai 2007 - I ZR 42/04, GRUR 2007, 691 Tz. 31 =WRP 2007, 996 Staatsgeschenk).

b) Unter Weiterveräußerung im Sinne des insoweit maßgeblichen deutschen Rechts ist jedenfalls die rechtsgeschäftliche Eigentumsübertragung, also das dingliche Verfügungsgeschäft, zu verstehen (BGHZ 126, 252, 259 - Folgerecht bei Auslandsbezug). Das Berufungsgericht hat angenommen, die Behauptung der Klägerin, die Einigung über den Eigentumsübergang sei schon in dem in Deutschland unterzeichneten Kaufvertrag enthalten gewesen, gelte als zugestanden, weil der Beklagte, den insoweit eine sekundäre Darlegungslast treffe, diese Behauptung nicht ausreichend substantiiert bestritten habe; demnach habe ein Teil des dinglichen Veräußerungsgeschäfts im Inland stattgefunden. Es kann dahinstehen, ob die gegen diese Beurteilung gerichteten Angriffe der Revision des Beklagten durchgreifen.

c) Der Begriff der Weiterveräußerung im Sinne des $\S$ 26 UrhG umfasst nicht allein das dingliche Verfügungsgeschäft, sondern das gesamte, aus dem schuldrechtlichen Verpflichtungs- und dem dinglichen Verfügungsgeschäft 
bestehende Veräußerungsgeschäft (Dreier/Schulze aaO § 26 Rn. 5; Schricker/ Katzenberger aaO Vor $\S 120$ ff. UrhG Rn. 146; Wandtke/Bullinger/v. Welser aaO Vor $\S 120 \mathrm{ff}$. UrhG Rn. 20; Braun IPRax 1995, 227, 229, 230; Schack JZ 1995, 357, 358 f.; v. Welser ZUM 2000, 472, 476 f.; Schneider-Brodtmann KUR 2004, 147, 152 f.; Katzenberger FS Schricker, 2005, S. 377, 382 ff.; vgl. auch Ulmer Urheber- und Verlagsrecht, 3. Aufl., S. 284; a.A. Katzenberger GRUR Int. 1992, 567, 582 f.; Vorpeil GRUR Int. 1992, 913 f.; Pfefferle GRUR 1996, 338, 340; Katzenberger Das Folgerecht im deutschen und ausländischen Urheberrecht, 1970, S. 94 f.; Schneider-Brodtmann Das Folgerecht des bildenden Künstlers im europäischen und internationalen Urheberrecht, 1996, S. 82 f.). Der Begriff der (Weiter-)Veräußerung schließt schuldvertragliche und sachenrechtliche Elemente ein (vgl. BGHZ 56, 256, 257 f. - Urheberfolgerecht) und legt daher eine Auslegung nahe, die sowohl den Kaufvertrag also auch die dingliche Verfügung umfasst. Im Übrigen begründet der Kaufvertrag die Zahlungsverpflichtung und bestimmt die Höhe des Veräußerungserlöses, an dem der Urheber nach § 26 UrhG zu beteiligen ist (Braun IPRax 1995, 227, 229; Schack JZ 1995, 357, 359; v. Welser ZUM 2000, 472, 476 f.). Allein das dingliche Verfügungsgeschäft vermag einen Folgerechtsanspruch deshalb ebenso wenig zu begründen wie das schuldrechtliche Verpflichtungsgeschäft. Der Begriff der Weiterveräußerung iSd § 26 UrhG schließt daher sowohl das dingliche als auch das schuldrechtliche Veräußerungsgeschäft ein. Demnach hat bereits mit der - unstreitigen - Unterzeichnung des Kaufvertrags durch die Verkäufer in Frankfurt am Main ein Teil der Weiterveräußerung im Inland stattgefunden. Damit liegt der für die Anwendung des § 26 UrhG erforderliche Inlandsbezug vor.

Dieses Ergebnis wird durch folgende Erwägung bestätigt: Das Folgerecht nach § 26 UrhG knüpft an das dem Urheber zustehende Verbreitungsrecht nach § 17 UrhG an. Ist das Original eines Werkes mit Zustimmung des zur Verbreitung Berechtigten gemäß § 17 Abs. 2 UrhG im Wege der Veräußerung in Verkehr gebracht worden, ist seine Weiterverbreitung mit Ausnahme der Vermietung zulässig. Aus dem Kreis der danach grundsätzlich freien Verbreitungshandlungen hat der Gesetzgeber die besondere Form der Weiterveräußerung, wie sie in $\S 26$ Abs. 1 Satz 1 UrhG a.F. geregelt ist, ausgenommen und mit einem abgeschwächten Vergütungsanspruch belastet (vgl. BGHZ 126, 252, 257 - Folgerecht bei Auslandsbezug). Für die Frage, welcher Teil der Weiterveräußerung für die Anwendung des § 26 UrhG im Inland erfolgt sein muss, kann daher darauf abgestellt werden, ob der fragliche Teilakt der (Weiter-)Veräußerung bereits den Tatbestand der Verbreitung i.S. des § 17 Abs. 1 UrhG erfüllt. Der Umstand, dass der Begriff der Verbreitung nach § 17 Abs. 1 UrhG sogar Vorbereitungshandlungen des Inverkehrbringens umfasst (vgl. Schulze in: Dreier/Schulze aaO § 17 Rn. 11 m.w.N.), macht deutlich, dass der im Inland erfolgte Abschluss des Kausalgeschäfts für eine Anwendung des § 26 UrhG ausreichend ist.

2. Der Anspruch des Urhebers gegen den Kunsthändler oder Versteigerer auf Auskunft über den Namen und die Anschrift des Veräußerers sowie über die Höhe des Veräußerungserlöses setzt nach $\S 26$ Abs. 4 Satz 1, Abs. 1 Satz 1 UrhG a.F. voraus, dass der Kunsthändler oder Versteigerer als Erwerber, Veräußerer oder Vermittler an der Weiterveräußerung des Originals eines Werkes der bildenden Künste beteiligt war. Das Berufungsgericht ist zwar - wie bereits oben unter B II 2 ausgeführt - zutreffend davon ausgegangen, dass der Beklagte als Kunsthändler iSv § 26 UrhG anzusehen ist. Die Feststellungen des Berufungsgerichts tragen jedoch nicht die Annahme, dass der Beklagte, wie das Berufungsgericht weiter angenommen hat, als Erwerber an der Veräußerung der "Sammlung Ahlers" beteiligt war.

a) Der Beklagte hat die Gemäldesammlung nach den Feststellungen des Berufungsgerichts gemeinsam mit einem amerikanischen Kunsthändler - seinem Partner N. - zum Zweck der Weiterveräußerung erworben. Das Berufungsgericht hat es dahingestellt sein lassen, ob die Behauptung des Beklagten zutrifft, Erwerber der Sammlung sei eine aus ihm und seinem Partner N. bestehende amerikanische Partnership gewesen. Im Revisionsverfahren ist daher zu unterstellen, dass der Beklagte und sein Partner die "Sammlung Ahlers" für die aus ihnen bestehende amerikanische Partnership zum Zweck der Weiterveräußerung erworben haben.

b) Die Revision des Beklagten rügt mit Erfolg, dass das Berufungsgericht das weitere Vorbringen des Beklagten zu dieser Partnership nicht berücksichtigt hat. Der Beklagte hat vorgetragen, bei dieser Partnership habe es sich um eine Partnership New Yorker Rechts gehandelt, die schon vor ihrer Eintragung als LLC (Limited Liability Company) rechtsfähig gewesen sei; diese Gesellschaft sei zum Erwerb von Vermögen im eigenen Namen befähigt gewesen; deren Gesellschafter würden selbst nicht Inhaber der von der Partnership erworbenen Vermögensstücke. Mangels entgegenstehender Feststellungen des Berufungsgerichts ist auch dieses Vorbringen des Beklagten in der Revisionsinstanz als zutreffend zugrunde zu legen. Dies gilt auch für die das amerikanische Recht betreffenden Rechtsbehauptungen des Beklagten. Der Tatrichter hat das in einem anderen Staat geltende Recht nach § 293 ZPO von Amts wegen zu ermitteln (BGHZ 153, 353, 358). Eine Verletzung dieser Ermittlungspflicht kann mit der Verfahrensrüge beanstandet werden (BGH, Urt. v. 23. April 2002 - XI ZR 136/01, NJW-RR 2002, 1359, 1360 mwN). Die Rechtsbehauptung des Beklagten, eine Partnership New Yorker Rechts sei bereits vor ihrer Eintragung als Limited Liability Company rechtsfähig, ist nach dem Recht der Vereinigten Staaten von Amerika - hier: nach dem Recht des Bundesstaates New York - zu beurteilen. Nach Art. XXV Abs. 5 Satz 2 des Freundschafts-, Handels- und Schiffahrtsvertrags zwischen der Bundesrepublik Deutschland und den Vereinigten Staaten von Amerika vom 29. Oktober 1954 (BGBI. 1956 II S. 487) gelten Gesellschaften, die gemäß den Gesetzen und sonstigen Vorschriften des einen Vertragsteils in dessen Gebiet errichtet sind, als Gesellschaften dieses Vertragsteils; ihr rechtlicher Status wird in dem Gebiet des anderen Vertragsteils als solcher anerkannt. Im Geltungsbereich dieses Abkommens ist das Personalstatut einer Gesellschaft somit an das am Ort ihrer Gründung geltende Recht anzuknüpfen. Das gilt auch 
hinsichtlich der Rechtsfähigkeit der Gesellschaft (BGH, Urt. v. 5. Juli 2004 - II ZR 389/02, NJW-RR 2004, 1618 mwN).

c) Ist demnach davon auszugehen, dass die "Sammlung Ahlers" von der rechtsfähigen D. \& N. Partnership erworben wurde, kann entgegen der Ansicht des Berufungsgerichts nicht angenommen werden, der Beklagte sei als Erwerber an der Veräußerung der "Sammlung Ahlers" beteiligt gewesen. Wird das Original eines Werkes der bildenden Künste bei einer Weiterveräußerung von einer rechtsfähigen Gesellschaft erworben, veräußert oder vermittelt, ist allein die rechtsfähige Gesellschaft an der Weiterveräußerung iSd § 26 Abs. 1 Satz 1 UrhG a.F. beteiligt, auch wenn die Gesellschafter dabei für die Gesellschaft handeln.

Das Berufungsgericht hat gemeint, es komme insoweit nicht auf die rechtliche Zuordnung des Eigentums an den Kunstgegenständen, sondern auf eine funktionelle Betrachtungsweise an, um Umgehungen des Folgerechtsanspruchs zu verhindern und dessen Durchsetzung im internationalen Kunsthandel nicht unangemessen zu erschweren. Deshalb müsse sich als Kunsthändler und Erwerber behandeln lassen, wer - wie der Beklagte - im internationalen Kunstgewerbe tätig sei und sich an einer Gesellschaft beteilige, die zum Zwecke des Erwerbs und der anschließenden Weiterveräußerung einer umfangreichen international angesehenen Kunstsammlung gegründet werde. Dem ist nicht zu folgen.

Die Befürchtung des Berufungsgerichts, die Durchsetzung von Folgerechtsansprüchen könne durch die Gründung einer Gesellschaft unangemessen erschwert werden, ist nicht begründet. Es gibt, wie die Revision des Beklagten zu Recht geltend macht, grundsätzlich keinen Grund, Gesellschaften für weniger geeignet zu halten, Folgerechtsansprüche zu erfüllen, als deren Gesellschafter. Es bestehen auch keine Anhaltspunkte dafür, dass die Durchsetzung des Auskunftsanspruchs im Streitfall durch die Gründung einer Gesellschaft erschwert wurde oder erschwert werden sollte. Es kann deshalb auch nicht als rechtsmissbräuchlich und daher unbeachtlich angesehen werden, dass der Beklagte sich darauf beruft, die "Sammlung Ahlers" nicht für sich selbst erworben zu haben und daher nicht auskunftspflichtig zu sein. Der Beklagte hat die Klägerin, nachdem er von ihr vorgerichtlich auf Auskunftserteilung in Anspruch genommen worden war, sogleich und wiederholt darauf hingewiesen, dass Erwerber der "Sammlung Ahlers" die D. \& N. Partnership sei und dass das Auskunftsersuchen deshalb an diese zu richten sei. Zugleich hat er deren Anschrift mitgeteilt. Die Klägerin hätte ihr Auskunftsersuchen daher ohne weiteres an diese Gesellschaft richten können.

3. Die Revision des Beklagten macht ohne Erfolg geltend, das Berufungsgericht habe die auf Verjährung des Hauptanspruchs gestützten Einwendungen des Beklagten übergangen und damit $\S 286,547$ Nr. 6 ZPO verletzt. Der Beklagte hat sich erstmals nach Schluss der mündlichen Verhandlung am 26. April 2005 in einem nicht nachgelassenen Schriftsatz vom 3. Juni 2005 darauf berufen, ein etwaiger Folgerechtsanspruch der Klägerin gegen die Ahlers AG aus $\S 26$ Abs. 1 UrhG sei verjährt. Das Berufungsgericht hat dieses Vorbringen zu Recht als verspätet zurückgewiesen und darin rechtsfehlerfrei keinen Anlass für eine Wiedereröffnung der Verhandlung gesehen (§ 525 Satz 1, §§ 296 a, 156 ZPO). Das Berufungsgericht hat sich daher entgegen der Ansicht der Revision des Beklagten zu Recht nicht mit der Frage auseinandergesetzt, ob einem Auskunftsanspruch gegen den Beklagten entgegensteht, dass dieser Auskunftsanspruch nicht zur Durchsetzung des Zahlungsanspruchs gegen den Veräußerer erforderlich ist (§ 26 Nr. 4 Satz 1 UrhG a.F.), weil dieser Zahlungsanspruch bereits verjährt ist und nicht mehr durchgesetzt werden kann (vgl. BGHZ 108, 393, 399). Im Übrigen hat das Berufungsgericht zutreffend darauf hingewiesen, dass an der Weiterveräußerung auf Seiten der Veräußerer neben der Ahlers AG jedenfalls noch weitere Unternehmen der Ahlers-Gruppe beteiligt waren. Es ist nicht ersichtlich, weshalb etwaige Zahlungsansprüche gegen diese - der Klägerin unbekannten - Veräußerer verjährt sein sollten.

C. Danach ist das Berufungsurteil auf die Revisionen der Parteien aufzuheben. Die Berufung des Beklagten gegen das dem allgemeinen Auskunftsanspruch stattgebende Urteil des Landgerichts ist zurückzuweisen. Hinsichtlich des die "Sammlung Ahlers" betreffenden Auskunftsanspruchs ist die Sache zur neuen Verhandlung und Entscheidung, auch über die Kosten des Revisionsverfahrens, an das Berufungsgericht zurückzuverweisen. Für die neue Verhandlung und Entscheidung wird auf Folgendes hingewiesen:

Das Berufungsgericht wird zu klären haben, ob die Behauptung des Beklagten zutrifft, dass die "Sammlung Ahlers" von der D. \& N. Partnership New Yorker Rechts vor deren Eintragung als Limited Liability Company erworben wurde; gegebenenfalls wird es ermitteln müssen, ob eine solche Partnership New Yorker Rechts schon vor ihrer Eintragung als Limited Liability Company rechtsfähig ist und ob allein die Gesellschaft Inhaber der von der Partnership erworbenen Vermögensgegenstände wird. Sollten diese Fragen zu bejahen sein, kommt es nicht weiter darauf an, ob die Gesellschafter einer Partnership New Yorker Rechts - BGB-Gesellschaftern vergleichbar (vgl. BGHZ 146, 341, 357) - für die Verbindlichkeiten der Gesellschaft haften, was gleichfalls nach amerikanischem Recht zu beurteilen wäre (vgl. BGH NJW-RR 2004, 1618), und ob die Klägerin den Beklagten danach auch wegen einer von der Gesellschaft geschuldeten Auskunftserteilung in Anspruch nehmen könnte (vgl. BGHZ 33, 302, 306). Die Klägerin nimmt den Beklagten im vorliegenden Rechtsstreit nicht wegen Verbindlichkeiten der Gesellschaft in Haftung. Sie verlangt von ihm Auskunftserteilung über die unter seiner Beteiligung als Erwerber, Veräußerer oder Vermittler veräußerten Kunstwerke der "Sammlung Ahlers" und nimmt ihn demnach ausschließlich wegen eigener Verbindlichkeiten in Anspruch. 